\title{
Efeitos dos Ritmos de Dança na Doença de Parkinson: Viabilidade de um Protocolo Para um Ensaio Clínico Randomizado
}

\section{Effects of Dance Rhythms on Parkinson's Disease: Feasibility of a Protocol for a Randomized Clinical Trial Effects of dance in Parkinson's disease}

\section{RESUMO}

Objetivo: Este estudo objetivou verificar a viabilidade de dois protocolos de dança - ritmo binário e ritmo quaternário nas variáveis UPDRS, equilíbrio, congelamento da marcha e qualidade de vida em indivíduos com doença de Parkinson. Metodologia: Realizou-se um ensaio clínico randomizado, comparando a viabilidade de dois protocolos de dança. Vinte sujeitos, diagnosticados com a doença de Parkinson, participaram do estudo e foram divididos aleatoriamente em dois grupos: grupo de ritmo binário e grupo de ritmo quaternário. A intervenção foi realizada duas vezes por semana, com duração de 45 minutos, durante 12 semanas. As variáveis analisadas foram: UPDRS, equilíbrio (Mini-BESTest), congelamento da marcha (FOG-Q) e qualidade de vida (PDQ-39). Resultados: Ambos os protocolos apresentaram resultados positivos no congelamento da marcha, UPDRS e qualidade de vida, além de mostrarem grande aderência nas intervenções, evidenciando serem importantes para o apoio no tratamento da doença. Conclusão: Este estudo de viabilidade é inovador em indivíduos com a doença de Parkinson, apresentando dois protocolos viáveis, que podem se tornar ferramentas relevantes para o tratamento de sintomas motores e não motores na doença de Parkinson.

\section{DESCRITORES}

Doença de Parkinson. Dança. Viabilidade. Protocolo.

\begin{abstract}
Objective: This study aimed to verify the feasibility of two dance protocols - binary rhythm and quaternary rhythm, in the variables UPDRS, balance, freezing of gait and quality of life in individuals with Parkinson's disease. Methodology: A randomized clinical trial comparing the feasibility of two dance protocols. Twenty subjects, diagnosed with Parkinson's disease, participated in the study and were randomly divided into two groups: binary rhythm group and quaternary rhythm group. The intervention was performed twice a week, lasting 45 minutes, for 12 weeks. The variables analyzed were: UPDRS, balance (Mini-BESTest), freezing of gait (FOG-Q) and quality of life (PDQ-39). Results: Both protocols showed positive results in freezing gait, UPDRS and quality of life, in addition to showing great adherence to interventions, showing that they are important to support the treatment of the disease. Conclusion: This feasibility study is innovative in individuals with Parkinson's disease, presenting two viable protocols, which can become relevant tools for the treatment of motor and non-motor symptoms in Parkinson's disease.
\end{abstract}

\section{DESCRIPTORS}

Parkinson's Disease. Dance. Viability. Protocol.

${ }^{1}$ Doutoranda em Ciências do Movimento Humano pelo Centro de Ciências da Saúde e do Esporte da Universidade do Estado de Santa Catarina, Florianópolis, Santa Catarina, Brasil.

${ }^{2}$ Mestranda em Ciências do Movimento Humano pelo Centro de Ciências da Saúde e do Esporte da Universidade do Estado de Santa Catarina, Florianópolis, Santa Catarina, Brasil.

${ }^{3}$ Professora Doutora do Departamento de Fisioterapia e do Programa de Pós-Graduação em Fisioterapia do Centro de Ciências da Saúde e do Esporte da Universidade do Estado de Santa Catarina, Florianópolis, Santa Catarina, Brasil.

${ }^{4}$ Professor Doutor do Departamento de Fisioterapia e do Programa de Pós-Graduação em Educação Física da Universidade Federal do Rio de Janeiro, Rio de Janeiro, Rio de Janeiro, Brasil.

${ }^{5}$ Doutora em Ciências do Movimento Humano pelo Centro de Ciências da Saúde e do Esporte da Universidade do Estado de Santa Catarina, Florianópolis, Santa Catarina, Brasil.

${ }^{6}$ Professora Doutora do Departamento de Educação Física e do Programa de Pós-Graduação em Ciências do Movimento Humano do Centro de Ciências da Saúde e do Esporte da Universidade do Estado de Santa Catarina, Florianópolis, Santa Catarina, Brasil. 
C om o envelhecimento da população, estima-se que em 2021, mais de 40 milhões de pessoas em todo o mundo apresentem a Doença de Parkinson (DP) ${ }^{1}$. Os acometidos por esta doença convivem durante anos com sintomas motores (bradicinesia, tremor de repouso, instabilidade postural, perda do equilíbrio e rigidez muscular) e não motores (distúrbios de memória, sintomas depressivos, fadiga excessiva, distúrbios do sono e redução na qualidade de vida) ${ }^{2,3}$. Estes sintomas podem estar relacionados ao aumento no número de quedas, gravidade da doença, hospitalização e a necessidade de cuidados ${ }^{4}$. Evidências recentes ressaltam a importância da dança como forma de atividade física benéfica e decisiva na melhoria do equilíbrio, mobilidade e em aspectos psicológicos nessa população, por meio de seus movimentos ritmados, variações rítmicas, mudanças direcionais espontâneas, alterações na velocidade, início e cessação dos movimentos ${ }^{5,6}$.

Desta forma, estudos de viabilidade de protocolos de dança são necessários e importantes, uma vez que reforçam a fixação e aderência obtidas no período de uma determinada intervenção de estudo ${ }^{7}$. Como a literatura mostra onde a viabilidade da prática de dança foi comparada com o caratê e com a fisioterapia convencional e mostraram que a dança teve maior adesão além de beneficiar os aspectos motores, sono, fadiga, bem-estar e o UPDRS ${ }^{8-10}$.

No entanto, deve-se questionar se esses resultados seriam os mesmos em diferentes modalidades de dança; qual seria a modalidade que poderia trazer maiores benefícios a essa população; a prática de qualquer tipo de dança irá gerar os mesmos resultados? Assim, optou-se por desenvolver dois protocolos de intervenção, separando as modalidades pelos seus respectivos ritmos, a fim de estudar não só as modalidades de dança, mas o próprio ritmo. Assim sendo, o ritmo binário caracterizado por um compasso de dois tempos e o ritmo quaternário, composto por um compasso de quatro tempos.

No protocolo de ritmo binário foram contempladas as modalidades de forró, merengue e bolero e no protocolo de ritmo quaternário o samba, salsa, zouk e tango. Acredita-se que o ritmo binário será bem aceito nesta população, uma vez que este ritmo contempla o forró, ritmo bastante conhecido e presente na cultura brasileira. Entretanto o protocolo de ritmo quaternário utiliza a modalidade samba, que também é muito popular entre os brasileiros, além da faixa etária a ser estudada demonstrar maior interesse nas modalidades realizadas com parceiro, por proporcionar maior segurança e proximidade com os colegas. Desta forma, o objetivo deste estudo foi verificar a viabilidade de dois protocolos - ritmo binário e ritmo quaternário nas variáveis UPDRS, equilíbrio, congelamento da marcha e qualidade de vida em indivíduos com a DP.

\section{METODOLOGIA}

O projeto foi aprovado pelo Comitê de Ética em Pesquisa em Seres Humanos (CEPSH) - da UDESC - Protocolo $n^{\circ}$. 2.380.719 e registrado no Clinical Trials $\mathrm{n}^{\circ}$. NCT03235453. O protocolo seguiu a declaração CONSORT sobre ensaios clínicos randomizados de tratamento não farmacológico e o SPIRIT ${ }^{11}$. 
O estudo de comparação da viabilidade de um protocolo de ritmo binário com um protocolo de ritmo quaternário, tendo ambos desenhos de ensaios clínicos randomizados, o qual procurou estabelecer relação de causa e efeito por meio da manipulação da variável dependente (ritmo binário e quaternário) sobre as variáveis independentes (UPDRS, equilíbrio, congelamento da marcha e qualidade de vida), realizando a avaliação antes e após a intervenção dos protocolos, tratando-se de dois grupos: a) grupo binário GB e b) grupo quaternário - GQ.

Desta forma participaram do estudo indivíduos com a DP idiopática de ambos os sexos, com média de idade $66,6 \pm 10,2$ anos, residentes em Santa Catarina - Brasil, que foram contatados por meio da Associação de Parkinson de Santa Catarina (APASC) e do Programa de Extensão Iniciativa Brasileira de Reabilitação na doença de Parkinson- BPaRkI do Centro de Ciências da Saúde e do Esporte (CEFID) da Universidade do Estado de Santa Catarina (UDESC). Os indivíduos foram divididos em dois grupos: a) GB que recebeu a intervenção do ritmo binário (forró, bolero e merengue): 10 indivíduos com média de idade de $68,3 \pm 8,6$ anos e b) GQ que recebeu a intervenção do ritmo quaternário (samba, zouk, tango e salsa): 10 indivíduos com média de idade de $64,3 \pm 14,8$ anos.

Como critérios de inclusão, foram incluídos indivíduos diagnosticados com DP idiopática clinicamente definida, como descrito nos critérios do banco de cérebro de Londres ${ }^{12}$, de ambos os sexos, com idade maior ou igual a 50 anos, que estavam na fase "On" da medicação e com doses estáveis há pelo menos 4 semanas, sem praticar nenhum tipo de dança há no mínimo três meses.

Foram excluídos aqueles que praticavam outra atividade física, não atingiram

Figura 1. Fluxograma de seleção dos participantes e execução das etapas do protocolo binário e quaternário

\section{CONSORT}

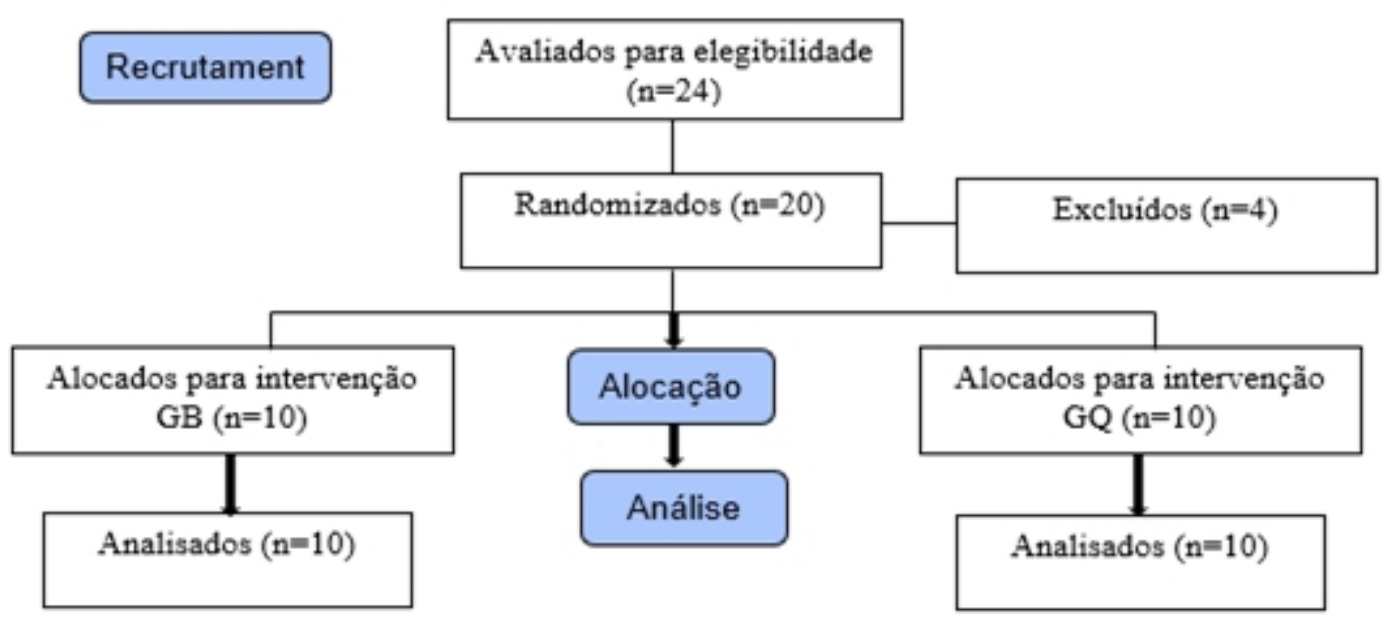


o ponto de corte do MEEM ${ }^{13}$ e que estiveram classificados no estágio 5 da DP ${ }^{14}$. Caso algum desses indivíduos iniciasse a prática regular de alguma outra atividade física neste período de 12 semanas, teriam seus dados coletados, porém seriam excluídos das intervenções. Da mesma forma, foram exclusos indivíduos que faltaram mais do que $25 \%$ da intervenção.

O fluxograma a seguir demonstra o processo de seleção dos participantes e execução das etapas do protocolo de estudo:

A randomização da amostra foi realizada por um pesquisador, com acesso à lista de indivíduos com DP. A partir dessa lista, realizou-se à randomização no programa de computador Microsoft Excel® de forma aleatória, que previu a distribuição dos mesmos nos dois grupos: GB - intervenção com ritmo binário e QG - intervenção com ritmo quaternário. Este pesquisador não fez parte da coleta de dados e nem da intervenção.

Com base no estudo de Cohen ${ }^{15} \mathrm{O}$ cálculo amostral foi realizado por meio do software G*Power 3.1.9.2, tamanho do efeito de 0,33 , nível de significância de $5 \%$ e poder do teste de $95 \%$ e perda amostral de $20 \%$. Dessa maneira esperou-se 20 indivíduos divididos entre o $G B$ e $G Q$.

A coleta de dados foi realizada por meio de questionário aplicado com entrevistas individuais pelos pesquisadores. Foram utilizados os seguintes instrumentos: Informações gerais; Escala Unificada de Avaliação da Doença de Parkinson (UPDRS) ${ }^{16}$; Teste de equilíbrio com o Mini-Bestest ${ }^{17}$; Congelamento da marcha com o Freezing of Gait - FOG-Q ${ }^{18}$; e qualidade de vida com o Parkinson's Disease Questionnaire - PDQ$39^{19}$.
Ambos os grupos realizaram a mesma metodologia de protocolo, a diferença foi o ritmo utilizado para cada protocolo (binário e quaternário).

Intervenção de ritmo binário

O ritmo binário, assim chamado, pois, consiste em um compasso de duas batidas (ex. dois passos para um lado e dois para o outro). As modalidades de dança contempladas neste protocolo que possuem ritmo binário foram o forró, o bolero e o merengue. Desta forma a aula foi dividida em: aquecimento (10 minutos) com caminhadas na marcação do ritmo musical, técnicas de liberação muscular nos membros inferiores e superiores, trabalhando a musicalidade a todo o momento. A parte principal (30 minutos) foi realizada por uma professora que mostrava e ensinava os passos a serem executados, em seguida os participantes realizavam os passos sozinhos e depois com seus parceiros. A cada 5 minutos os participantes trocavam de parceiros, para que todos pudessem ter outras experiências ao dançar com seus colegas, além de estimular a liderança. Por fim, alongamento e caminhada lenta (período de descanso), realizados por 5 minutos para proporcionar relaxamento muscular.

Os movimentos de dança foram realizados em diferentes intensidades (leve, moderada e vigorosa) para que os indivíduos tivessem uma progressão ao longo das 12 semanas. A pressão arterial (PA) e a frequência cardíaca (FC) foram monitoradas durante as aulas para maior segurança dos participantes durante as intervenções e nos aumentos das intensidades. 
Quadro 1. Descrição dos passos ministrados no protocolo de ritmo binário

\begin{tabular}{|c|c|}
\hline Passos & Caracterização \\
\hline $\begin{array}{l}\text { Apresentação do ritmo } \\
\text { binário }\end{array}$ & $\begin{array}{l}\text { Durante a aula são executados movimentos de deslocamento com músicas de } \\
\text { diferentes velocidades para ambientar os alunos ao ritmo. }\end{array}$ \\
\hline $\begin{array}{c}\text { Dois para lá, dois para } \\
\text { cá }\end{array}$ & $\begin{array}{l}\text { Caracterizado por dois passos para um lado e voltando dando dois passos para } \\
\text { o outro lado, transferindo o peso do corpo. }\end{array}$ \\
\hline Caminhada & $\begin{array}{c}\text { Caminhada para frente e voltando para trás (dois passos para frente e dois para } \\
\text { trás). }\end{array}$ \\
\hline Dobradiça & $\begin{array}{l}\text { Abertura lateral como uma porta, a dama ficará de frente para o cavalheiro com } \\
\text { as mãos dadas, soltarão uma das mãos e darão um passo para a lateral, após } \\
\text { voltarão para a posição inicial. }\end{array}$ \\
\hline Fixação & $\begin{array}{l}\text { Realizar o passo dois para lá dois para cá, caminhada e dobradiça em diferentes } \\
\text { intensidades e velocidades da música. }\end{array}$ \\
\hline Giro simples & $\begin{array}{l}\text { Saindo do passo básico o cavalheiro conduzirá a dama para realizar o giro } \\
\text { simples, a dama realizará o giro deslocando para frente e o cavalheiro } \\
\text { acompanhará seu movimento caminhando para frente ao seu lado. }\end{array}$ \\
\hline Trocadilho & $\begin{array}{c}\text { A dama e o cavalheiro caminharão para o lado cruzando as pernas (cruza, abre) } \\
\text { e voltarão para o outro lado da mesma forma. }\end{array}$ \\
\hline Chuveirinho & $\begin{array}{c}\text { A dama ficará de frente para o cavalheiro com as mãos dadas, ambos subirão os } \\
\text { dois braços e a dama realizará meio giro no lugar ficando de costas para o } \\
\text { cavalheiro, assim, dará outro meio qiro, voltando na posicão inicial. }\end{array}$ \\
\hline Leque simples & $\begin{array}{l}\text { A dama e o cavalheiro ficarão de lado com as mãos dadas, irão caminhar para } \\
\text { frente e trás com a mesma perna. }\end{array}$ \\
\hline Giro duplo & $\begin{array}{l}\text { A dama e o cavalheiro ficarão de frente um para o outro com as mãos dadas, os } \\
\text { dois irão caminhar para o lado esquerdo, soltar as mãos e realizar um giro no } \\
\text { lugar. }\end{array}$ \\
\hline Revisão & $\begin{array}{l}\text { Os alunos realizarão uma revisão de todos os passos ensinados na aula, para } \\
\text { fixação e reforçar o que foi aprendido. }\end{array}$ \\
\hline Roda de dança & $\begin{array}{c}\text { Livremente os alunos dançarão entre si trocando de par, executando os passos } \\
\text { aprendidos em forma de baile. }\end{array}$ \\
\hline
\end{tabular}

Intervenção de ritmo quaternário

$$
\text { O ritmo quaternário é caracterizado }
$$

por um compasso formado por quatro batidas (quatro tempos) que possui grande diversidade de execução. As modalidades utilizadas com o ritmo quaternário neste grupo foram o samba, o tango, a salsa e o zouk. A intervenção foi realizada seguindo a intensidade (leve, moderada e vigorosa). A pressão arterial (PA) e a frequência cardíaca (FC) foram monitoradas durante as aulas para maior segurança dos participantes durante as intervenções e nos aumentos das intensidades.

Todas as aulas tiveram duração de 45 minutos, organizadas: aquecimento focado em estilos de dança que incluem o ritmo quaternário, com marcações de passos rítmicos, liberação muscular, respiração, enquanto estimula constantemente a musicalidade. A parte principal onde o professor demonstrou os passos a serem desenvolvidos durante a aula. Posteriormente, os alunos repetiram o mesmo movimento em pares, os pares mudavam constantemente. Durante 
o período de volta à calma, os indivíduos realizaram alongamentos e caminhada lenta para proporcionar relaxamento muscular.

Análise estatística

Os dados foram analisados por meio do pacote estatístico SPSS - IBM, versão 20.0, no qual foram realizadas estatísticas descritivas e inferenciais. A comparação foi realizada por meio da Anova two way com medidas repetidas e teste de comparação de Sydak entre os grupos GB e GQ no período pré e pós-intervenção, nas variáveis: UPDRS, equilíbrio, congelamento da marcha e qualidade de vida. O teste exato de Fisher foi usado para informações gerais. Nível de significância adotado de 5\%.

\section{RESULTADOS}

A Tabela 1 apresenta a caracterização dos participantes segundo o grupo binário e quaternário, apresentando grupos homogêneos, sem diferenças significativas entre eles.

\section{Quadro 2. Descrição dos passos ministrados no protocolo de ritmo quaternário}

\begin{tabular}{|l|l|}
\hline \multicolumn{1}{|c|}{ Passos } & \multicolumn{1}{c|}{ Caracterização } \\
\hline $\begin{array}{l}\text { Apresentação do ritmo } \\
\text { quaternário }\end{array}$ & $\begin{array}{l}\text { Durante a aula são executados movimentos de deslocamento com músicas de } \\
\text { diferentes velocidades para ambientar os alunos ao ritmo. }\end{array}$ \\
\hline $\begin{array}{l}\text { Deslocamento com } \\
\text { marcação do ritmo }\end{array}$ & Realizar uma caminhada em 4 tempos para frente para trás no ritmo lento. \\
\hline Passo básico frente/trás & Caracterizado por um passo à frente, dois passos no lugar e um passo atrás. \\
\hline Passo básico lateral & $\begin{array}{l}\text { Caracterizado por um passo ao lado, dois passos no lugar e um passo em } \\
\text { retorno à posição inicial. }\end{array}$ \\
\hline $\begin{array}{l}\text { Passo básico frente/trás } \\
\text { e lateral }\end{array}$ & $\begin{array}{l}\text { Efetuar a junção dos dois passos anteriormente aprendidos, caracterizados por } \\
\text { frente/trás/lado/lado. }\end{array}$ \\
\hline Giro no lugar & $\begin{array}{l}\text { Lado a lado, dama e cavalheiro partem do passo básico frente/trás para um giro } \\
\text { em torno do próprio eixo, marcando com o mesmo pé à frente e atrás após o } \\
\text { qiro. }\end{array}$ \\
\hline Giro da dama & $\begin{array}{l}\text { Saindo do passo básico para trás, a dama executa um giro após marcar o passo } \\
\text { à frente em torno do próprio eixo, o braço deve estar acima do corpo com o } \\
\text { auxilio do cavalheiro. }\end{array}$ \\
\hline Condução & $\begin{array}{l}\text { Saindo do passo básico, cavalheiro deve passar o braço em volta da cintura da } \\
\text { dama deixando-a ao seu lado e conduzi-la ao outro lado. }\end{array}$ \\
\hline $\begin{array}{l}\text { Giro da dama com } \\
\text { deslocamento }\end{array}$ & $\begin{array}{l}\text { Saindo do passo básico, cavalheiro dá um passo para o lado de forma com que } \\
\text { a dama fique ao seu lado, dama afasta-se deixando o braço dos dois estendidos } \\
\text { e retorna enrolando o braço do cavalheiro no próprio corpo. }\end{array}$ \\
\hline Sequência de passos & $\begin{array}{l}\text { Os alunos repetem os passos aprendidos um após o outro, inicialmente de } \\
\text { maneira pausada até conseguirem realizá-los na música. }\end{array}$ \\
\hline Revisão dos passos & $\begin{array}{l}\text { Será realizada uma revisão de todos os passos aprendidos, a fim de reforçar o } \\
\text { aprendizado. } \\
\text { cavalheiro, cruzando em quatro tempos um pé por vez. }\end{array}$ \\
\hline & $\begin{array}{l}\text { Dama, com o auxílio do cavalheiro, atravessa os pés em direção aos do } \\
\text { Cruzada }\end{array}$ \\
\hline
\end{tabular}


Tabela 1. Caracterização dos participantes do estudo de acordo com o grupo binário e quaternário

\begin{tabular}{|c|c|c|c|}
\hline & $n(\%)$ & $n(\%)$ & $p^{*}$ \\
\hline Sexo & & & 1,000 \\
\hline Masculino & $07(70)$ & $07(70)$ & \\
\hline Feminino & $03(30)$ & $03(30)$ & \\
\hline Escolaridade & & & 0,066 \\
\hline Ensino Fundamental & $05(50)$ & $06(60)$ & \\
\hline Ensino Médio Completo & $05(50)$ & $002(20)$ & \\
\hline Ensino Superior & - & $2(20)$ & \\
\hline Estado Conjugal & & & 1,000 \\
\hline Com Companheiro & $07(70)$ & $07(70)$ & \\
\hline Sem Companheiro & $03(30)$ & $03(30)$ & \\
\hline Doenças Concomitantes & & & 0,500 \\
\hline Não & $05(50)$ & $04(40)$ & \\
\hline Sim & $05(50)$ & $06(60)$ & \\
\hline Manifestação Inicial & & & 0,700 \\
\hline Tremor & $06(60)$ & $05(50)$ & \\
\hline Bradicinesia & $01(10)$ & $01(10)$ & \\
\hline Rigidez & $01(10)$ & $03(30)$ & \\
\hline Marcha & $02(20)$ & $01(10)$ & \\
\hline Remédio para dormir & & & 0,531 \\
\hline Não & $09(90)$ & $08(80)$ & \\
\hline Sim & $01(10)$ & $02(20)$ & \\
\hline Outros medicamentos & & & \\
\hline Não & - & $01(10)$ & 0,305 \\
\hline Sim & $10(100)$ & $09(90)$ & \\
\hline
\end{tabular}

$\mathrm{Na}$ Tabela 2 são apresentados os resultados relacionados ao UPDRS, equilíbrio e congelamento da marcha. Está evidenciado que não existiram diferenças significativas entre os grupos quando se comparou pós com pós entre as variáveis, porém, ocorreram diferenças entre o grupo pré e pós tanto no GB como no GQ no congelamento da marcha $(p=0,022 ; p=0,046)$, respectivamente, assim como em algumas variáveis do UPDRS em ambos os grupos, nomeadamente, atividades da vida diária $(p=0,017 ; p=0,049)$, exame motor ( $p=0,008 ; p=0,002)$ e complicações na terapia $(p=0,010 ; p=0,013)$, respectivamente. O equilíbrio obteve significância apenas no GQ quando comparado pré com pós intervenção $(p=0,016)$.

De acordo com a Tabela 3 , apresentados os resultados relacionados à qualidade de vida, salienta-se a ausência de diferença significativa entre os grupos quando comparado pós com pós entre as variáveis. Porém, ocorreram diferenças significativas entre o grupo pré e pós tanto no GB como no $G Q$ na variável mobilidade $(p=0,030 ; p=0,002)$, emocional $(p=0,001)$ e $G Q(p=0,001)$, cognição $(p=0,039)$ e $G Q(p=0,008)$ desconforto $(p=0,017)$ e $G Q(p=0,022)$ e na qualidade de vida total $(p=0,001)$ e $G Q(p=<0,001)$. O GB também apresentou diferença significante na variável de atividade diária $(p=0,022)$ e o GQ na variável comunicação $(p=0,018)$.

Aspectos da viabilidade

Durante a intervenção, não ocorreu 
Tabela 2. Escores do UPDRS, Equilíbrio e Congelamento da Marcha dos participantes de acordo com o grupo

\begin{tabular}{|c|c|c|c|c|c|c|c|c|c|}
\hline & \multicolumn{3}{|c|}{ GB } & \multicolumn{3}{|c|}{ GQ } & \multicolumn{3}{|c|}{ Pós } \\
\hline & Pré & Pós & & & Pré & Pós & & & \\
\hline & $\dot{X}(d p)$ & $\dot{X}(d p)$ & $\mathrm{CE}$ & $\mathrm{P}^{*}$ & $\dot{\mathrm{X}}(\mathrm{dp})$ & $\dot{X}(d p)$ & $\mathrm{CE}$ & $\mathrm{P}^{*}$ & $\mathrm{P \#}$ \\
\hline UPDRS & & & & & & & & & \\
\hline Estado Mental & $2,8(1,8)$ & $2,1(1,6)$ & 0,7 & 0,053 & $2,9(2,0)$ & $2,3(1,5)$ & 0,6 & 0,092 & 0,785 \\
\hline Atividades Diárias & $13,2(7,8)$ & $9,7(5,7)$ & 3,5 & 0,017 & $16,0(11,6)$ & $13,2(7,8)$ & 2,8 & 0,049 & 0,272 \\
\hline Exame Motor & $12,9(9,6)$ & $9,0(7,3)$ & 3,9 & 0,008 & $18,5(12,2)$ & $13,9(9,1)$ & 4,6 & 0,002 & 0,204 \\
\hline $\begin{array}{l}\text { Complicações na } \\
\text { Terapia }\end{array}$ & $7,1(4,3)$ & $4,9(3,2)$ & 2,2 & 0,010 & $6,0(4,0)$ & $3,9(2,9)$ & 2,1 & 0,013 & 0,478 \\
\hline $\begin{array}{l}\text { Equilíbrio } \\
\text { Congelamento da }\end{array}$ & $\begin{array}{c}19,5(5,6) \\
8,60(7,01)\end{array}$ & $\begin{array}{c}20,6(5,7) \\
5,90(4,65)\end{array}$ & $\begin{array}{r}-1,1 \\
2,7\end{array}$ & $\begin{array}{l}0,292 \\
0,022\end{array}$ & $\begin{array}{l}17,8(5,5) \\
10,0(5,37)\end{array}$ & $\begin{array}{c}20,5(5,3) \\
7,70(4,64)\end{array}$ & $\begin{array}{r}-5,7 \\
2,3\end{array}$ & $\begin{array}{l}0,016 \\
0,046\end{array}$ & $\begin{array}{l}0,968 \\
0,398\end{array}$ \\
\hline
\end{tabular}

$\mathrm{X}=$ média; $\mathrm{dp}=$ Desvio Padrão; UPDRS= Escala Unificada de Avaliação para a Doença de Parkinson. " $\mathrm{p}$ valor para comparação entre os períodos pré e pós do GB e GQ; \# p valor para comparação entre os GB e GQ no período pós intervenção. Utilizou-se o Teste Anova two way com medidas repetidas e Teste de comparação de Sydak; CE= Chance escores.

Tabela 3. Percepção de qualidade de vida de indivíduos com a Doença de Parkinson

\begin{tabular}{|c|c|c|c|c|c|c|c|c|c|}
\hline & \multicolumn{3}{|c|}{ GB } & \multicolumn{5}{|c|}{ GQ } & \multirow[t]{2}{*}{ Pós } \\
\hline & Pré & Pós & & & Pré & Pós & & & \\
\hline Qualidade de Vida & $\dot{\mathrm{X}}(\mathrm{dp})$ & $\dot{\bar{X}}(\mathrm{dp})$ & $\mathrm{CE}$ & $P^{*}$ & $\dot{\mathrm{X}}(\mathrm{dp})$ & $\dot{X}(d p)$ & $\mathrm{CE}$ & $\mathrm{P}^{*}$ & $\mathrm{P \#}$ \\
\hline Mobilidade & $16,2(12,0)$ & $13,1(12,3)$ & 3,1 & 0,030 & $13,4(10,6)$ & $8,5(8,0)$ & 4,9 & 0,002 & 0,337 \\
\hline Atividade Diária & $9,5(8,0)$ & $6,0(5,9)$ & 3,5 & 0,022 & $9,0(7,8)$ & $6,2(6,5)$ & 2,8 & 0,061 & 0,994 \\
\hline Emocional & $8,6(5,8)$ & $4,8(4,8)$ & 3,8 & 0,001 & $8,3(5,9)$ & $4,1(4,9)$ & 4,2 & 0,001 & 0,753 \\
\hline Estigma & $2,8(3,0)$ & $2,4(4,5)$ & 0,4 & 0,664 & $3,6(4,7)$ & $2,5(4,1)$ & 1,1 & 0,240 & 0,959 \\
\hline Suporte & $8,0(-)$ & $7,7(0,4)$ & 0,3 & 0,232 & $7,5(1,3)$ & $8,0(0,94)$ & -0.5 & 0,054 & 0,382 \\
\hline Cognição & $5,7(5,1)$ & $3,4(3,1)$ & 2,3 & 0,039 & $7,1(3,8)$ & $4,0(2,1)$ & 3,1 & 0,008 & 0,624 \\
\hline Comunicação & $3,4(3,5)$ & $2,7(3,1)$ & 0,7 & 0,271 & $3,3(2,9)$ & $1,7(2,7)$ & 1,6 & 0,018 & 0,463 \\
\hline Desconforto & $6,4(4,5)$ & $4,0(2,4)$ & 2,4 & 0,017 & $5,0(2,1)$ & $2,7(1,5)$ & 2,3 & 0,022 & 0,169 \\
\hline Total & $59.6(32.5)$ & $44,1(29,9)$ & 15.5 & 0.001 & $56.9(29.8)$ & $37.7(24,1)$ & 19.2 & $<0.001$ & 0.605 \\
\hline
\end{tabular}

$\mathrm{X}=$ média; $\mathrm{dp}=$ Desvio Padrão; UPDRS= Escala Unificada de Avaliação para a Doença de Parkinson. ${ }^{*} \mathrm{p}$ valor para comparação entre os períodos pré e pós do $\mathrm{GB}$ e GQ; \# p valor para comparação entre os GB e GQ no período pós intervençãol Utilizou-se o Teste Anova two way com medidas repetidas e Teste de comparação de Sydak; CE= Chance Escore.

episódio de queda; acreditando-se que a atenção dada pelos pesquisadores durante as sessões foi de grande importância para orientar os participantes que apresentavam alguma dificuldade na execução do movimento, bem como mantê-los interessados e motivados, sempre enfatizando o melhor do participante. Todas as intervenções foram bem-sucedidas conforme previsto no protocolo; não foi verificada a ocorrência que mostrasse que alguma atividade era inadequada. Isso sugere que os participantes conseguiram realizar as tarefas propostas mesmo com complexidade e intensidade crescentes.

\section{Aderência}

A aderência às intervenções de ritmo binário e quaternário foi estabelecida, com um resultado de $84,3 \%$ de sessões prescritas no protocolo cumprido, conforme o estudo de aderência de Allen et al. ${ }^{20}$. Após doze semanas, não se observou casos de desistência, o que reforça nossa hipótese de que o protocolo foi útil para melhorar a adesão do paciente a um programa de reabilitação. 


\section{DISCUSSÃO}

Este estudo de viabilidade é o primeiro a comparar um protocolo de ritmo binário com um protocolo de ritmo quaternário nas variáveis UPDRS, equilíbrio, congelamento da marcha e qualidade de vida em indivíduos com DP. Os dois protocolos mostraram-se viáveis com alto nível de adesão (84,3\%), durante as 12 semanas de intervenção. Além dos resultados estatísticos, as características como a disposição dos indivíduos em serem randomizados, sucesso da randomização, recursos necessários, custos, aplicabilidade, progressão da intervenção, aceitabilidade e segurança dos participantes durante as intervenções também contribuíram para que esses protocolos fossem considerados viáveis.

Desta forma os resultados encontrados no estudo mostraram diferenças de significância intragrupo na maioria das variáveis estudadas; mas não ocorreram diferenças significativas intergrupos. Ainda assim, este estudo mostrou que um período de 12 semanas produziu melhorias nas variáveis de forma eficaz, confirmando a eficácia dos dois protocolos utilizados separadamente. Esses resultados estão de acordo com outros estudos, nos quais intervenções rítmicas foram utilizadas em indivíduos com DP ${ }^{21-23}$.

No UPDRS, ambos os grupos apresentaram melhora em três das quatro avaliações, sendo atividades diárias, exame motor e complicações na terapia. Nota-se que com o passar do tempo a DP faz com que o desempenho das atividades diárias do indivíduo piore gradativamente, pois os movimentos que estas requerem podem ganhar dificuldade de execução causada no decorrer da doença, afetando outras capacidades, por isto, um bom desempenho das atividades diárias é fundamental para a preservação de todas as suas atividades mentais e motoras ${ }^{24}$. Logo, sugere-se que a melhora dos resultados das atividades diárias pode estar relacionada à melhora do estado motor assim como nas complicações na terapia, pois os protocolos de ritmo binário e quaternário foram desenvolvidos com passos direcionados às capacidades mais afetadas pela DP, como passos básicos de deslocamento que ganharam maiores dificuldades de execução ao decorrer das semanas, assim como giros tanto individuais, como com auxílio e até mesmo giros sincronizados com o parceiro. Estes movimentos podem ter auxiliado na execução dos movimentos básicos necessários para as atividades diárias o que possivelmente afetou de forma positiva as outras avaliações.

Ainda, outro sintoma que afeta diretamente a vida dos indivíduos com DPé a perda do equilíbrio. Um bom nível de equilíbrio é um fator essencial para todos os indivíduos, principalmente para aqueles com DP, uma vez que o desequilíbrio pode ser sinônimo de quedas ${ }^{25}$. Na variável equilíbrio, apenas o GQ apresentou resultados significativos. Os resultados corroboram com intervenções de estudos anteriores ${ }^{26,27}$ realizados com o tango, o que nos leva à hipótese de que intervenções com ritmo quaternário podem ter influência direta no nível de equilíbrio por conta dos exercícios de deslocamento, variações nas direções como para frente, para trás, direita, esquerda e ainda alternando as diferentes direções ou com dupla tarefa. Além de que 
passos como giro duplo e giro no lugar podem ter contribuído para esse achado.

Assim como a perda de equilíbrio, o congelamento da marcha também é um problema frustrante na DP, que consiste em uma redução na progressão à frente dos pés apesar da intenção de caminhar ${ }^{28-30}$. Dos Santos Delabary et al. ${ }^{31}$, em uma revisão sistemática com metanálise, mostram melhora nos resultados do congelamento da marcha em intervenções de dança em comparação a outros tipos de exercícios físicos, o que está de acordo com os resultados obtidos neste estudo, que evidenciou que a utilização de diferentes ritmos de dança auxiliam na melhora do congelamento da marcha, pois, trabalha com mudanças de direções, passos pequenos, médios e longos, com paradas bruscas, além de exigir que o indivíduo seja capaz de girar e sincronizar esse movimento com o movimento do parceiro ${ }^{28}$.

Além dos sintomas motores mencionados, a DP também reduz a qualidade de vida. Assim, a dança é uma alternativa viável, pois atua diretamente na melhora do dos sintomas, conforme apresentado em outros estudos ${ }^{21,22,31}$. Os grupos de intervenção binário e quaternário apresentaram melhora significativa em cinco das oito variáveis de qualidade de vida. $A$ mobilidade apresentou melhora significativa em ambos os grupos, assim como emocional, cognitiva e desconforto. Além desses, o GB também apresentou resultados significativos nas atividades diárias e o GQ na comunicação. Dahmen-Zimmer e Jansen ${ }^{9}$ relacionaram a dança (valsa e rumba) com o emocional e apresentaram resultados positivos após as intervenções, corroborando os achados do presente estudo.

Kalyani et al. ${ }^{21}$ compararam em seu estudo os efeitos de uma intervenção de dança para indivíduos com DP e observaram que o grupo que executou dança apresentou melhores resultados na cognição, ansiedade, depressão e qualidade de vida em comparação ao grupo controle. Michels et al. ${ }^{32}$ realizaram em seu estudo uma comparação entre um grupo com intervenção de dança terapia e um grupo de controle, notou-se que os indivíduos que participaram das aulas de dança terapia melhoraram a cognição entre outros aspectos motores e não motores. Podemos destacar a partir dos resultados do presente estudo que diferentes modalidades de dança podem atuar para melhorar a cognição. Acredita-se que aprender novos passos a cada aula, memorizando-os e dificultando-os a cada semana, relacionando-os às suas respectivas canções, pode estimular o desenvolvimento da cognição, obtendo assim excelentes resultados em ambos os grupos ${ }^{32,33}$.

A proposta da dança como atividade física para indivíduos com DP da qual se tratam os protocolos em questão, mostraramse predominantemente como atividades que exigem uma gama de habilidades que são distintas a resistência aeróbica ${ }^{34,35}$. Petzinger et al. ${ }^{36}$ revelaram evidências recentes que confirmam que o tipo de exercício pode ter efeitos sobre os circuitos cerebrais, como exercícios que requerem diferentes habilidades afetando diferentes áreas do cérebro, tal qual os circuitos relacionados ao estriado frontal em um grau mais alto do que o exercício aeróbio puro. $\mathrm{O}$ autor sugere ainda que diferentes tipos de exercícios sobre a neuroplasticidade e a melhora motora e cognitiva na DP podem fornecer informações que são essenciais para fornecer o reparo do cérebro e estabilizar a progressão da doença.

Ainda neste estudo, a aceitação dos 
indivíduos para serem randomizados foi efetiva e não ocorreram problemas neste processo, o que facilitou o sucesso da randomização, visto que ambos os grupos possuíam participantes com níveis semelhantes de DP. Os recursos necessários para aplicar esses protocolos são simples, exigindo apenas professor, espaço e aparelho de som, tornando o custo baixo ou praticamente nulo; as aulas foram oferecidas gratuitamente aos participantes. Os protocolos apresentaram aceitabilidade, onde todos realizaram o que foi solicitado nas intervenções e foram aplicáveis, pois em nenhuma atividade foram necessárias alterações. As intervenções foram progressivas, pois, seguiram a sugestão de aumento da complexidade das etapas de forma gradativa e aumento da intensidade e foram seguras por não ter ocorrido episódio de queda.

\section{CONCLUSÃO}

Com base nos resultados apresentados, conclui-se que tanto a aplicação dos protocolos de ritmo binário quanto ritmo quaternário são viáveis para indivíduos com DP, apresentandose como atividades seguras, prazerosas e com benefícios motores e não motores satisfatórios, tornando esta atividade uma alternativa de terapia não farmacológica que pode ser utilizada de forma simultânea ao tratamento farmacológico. Ambos os protocolos podem ser facilmente implantados em qualquer lugar do mundo. Intervenções baratas, acessíveis e não invasivas que abordem sintomas motores e não motores na DP são de suma importância para estes indivíduos, estes protocolos são promissores como alternativas úteis no tratamento dos sintomas da DP.

\section{REFERENCIAS}

1. Jagadeesan AJ, Murugesan R, Devi SV, et al. Current trends in etiology, prognosis and therapeutic aspects of Parkinson's disease: a review. Acta Biomed. 2017;88(3):249-262.

2. Cabreira V, Massano J. Parkinson's Disease: Clinical Review and Update. Acta Med Port. 2019;32(10):661-670.

3. Balestrino R, Schapira AH. Parkinson Disease. European journal of neurology. 2019; 27(1):27-42.

4. Pelicioni PHS, Menant JC, Latt MD, Lord SR. Falls in Parkinson's Disease Subtypes: Risk Factors, Locations and Circumstances. Int $\mathrm{J}$ Environ Res Public Health 2019; 23(16):2216.

5. Kalyani HH, Sullivan KA, Moyle GM, Brauer S, Jeffrey ER, Kerr GK. Dance improves symptoms, functional mobility and fine manual dexterity in people with Parkinson disease: a quasi-experimental controlled efficacy study. Eur J Phys Rehabil Med. 2020; 56 (5):563-574.

6. Kalyani HHN, Sullivan K, Moyle G, et al. Effects of Dance on Gait, Cognition, and Dual-Tasking in Parkinson's Disease: A Systematic Review and Meta-Analysis. J Parkinsons Dis. 2019;9(2):335-349.

7. Lihala S, Mitra S, Neogy S, Datta N, et al. Dance movement therapy in rehabilitation of Parkinson's disease - A feasibility study. J Bodyw Mov Ther. 2021; 26:12-17

8. Shanahah J, Morris ME, Bhriain ON, Volpe D, Richardson M, Clifford AM. "Is Irish set dancing feasible for people with Parkinson's disease in Ireland?" Complementary therapies in clinical practice. 2015;21(1):47-51.

9. Dahmen-Zimmer K, Jansen P. Karate and Dance Training to Improve Balance and Stabilize Mood in Patients with Parkinson's Disease: A Feasibility Study. Front Med (Lausanne). 2017;4:237

10. Tillmann AC, Swarowsky A, Corrêa CL, et al. Feasibility of a Brazilian samba protocol for patients with Parkinson's disease: a clinical non-randomized study. Arq Neuropsiquiatr. 2020;78(1):13-20.

11. Chan AW, Tetzlaff JM, Altman DG, Laupacis A, Gøtzsche PC, Krleža-Jerić K, et al. SPIRIT 2013 statement: defining standard protocol items for clinical trials. Ann Intern Med. 2013;158:200-207

12. Hughes AJ, Daniel SE, Kilford L, Lees AJ. Accuracy of clinical diagnosis of idiopathic Parkinson's disease: a clinico-pathological study of 100 cases. J Neurol Neurosurg Psychiatry. 1992;55(3):181-184.

13. Bertolucci PHF, Brucki SMD, Campacci SR, Juliano Y. O Mini-Exame do Estado Mental em uma população geral: impacto da escolaridade. Arq. NeuroPsiquiatr.1994;52(1):01-07. 
14. Hoehn MM, Yahr MD. Parkinsonism: onset, progression and mortality. Neurology. 1967;17(5):427-442

15. Cohen J. "Statistical power analysis for the behavioural sciences" Hillsdale: Lawrence Erbaum Associates, 2.a ed, 1988.

16. Movement Disorder Society Task Force on Rating Scales for Parkinson's disease. "The Unified Parkinson's Disease Rating Scale (UPDRS): status and recommendations." Movement disorders: official journal of the Movement Disorder Society, 2003;18(7):738.

17. Maia AC, Rodrigues-de-Paula F, Magalhães LC, Teixeira RLL. Cross-cultural adaptation and analysis of the psychometric properties of the Balance Evaluation Systems Test and MiniBESTest in the elderly and individuals with Parkinson's disease: application of the Rasch model. Braz. J. Phys. Ther. 2013;17(3):195-217.

18. Baggio JAO, Curtarelli MB, Rodrigues GR, Tumas V. Validity of the Brazilian version of the freezing of gait questionnaire. Arq. Neuro-Psiquiatr. 2012; 70(8):599-603.

19. Souza RG, Borges V, Silva SMCA, Ferraz HB. Quality of life scale in parkinson's disease PDQ-39 - (Brazilian Portuguese version) to assess patients with and without levodopa motor fluctuation. Arq. Neuro-Psiquiatr. 2007; 65(3b):787-791.

20. Allen NE. Song J, Sherrington CC, Lord SR, Fung VSC, Close JCT, Paul SS, O'rourke SD, Murray SM, Canning SG. Predictors of adherence to an exercise program in people with Parkinson's disease. Movement Disorders 2013; 28(1):440.

21. Kalyani HHN, Sullivan KA, Moyle G, et al. Impacts of dance on cognition, psychological symptoms and quality of life in Parkinson's disease. NeuroRehabilitation. 2019; 45(2):273-283.

22. Solla $\mathrm{P}$, Cugusi L, Bertoli M, et al. Sardinian Folk Dance for Individuals with Parkinson's Disease: A Randomized Controlled Pilot Trial. J Altern Complement Med. 2019; 25(3):305-316.

23. Pereira APS, Marinho V, Gupta D, Magalhães F, Ayres C, Teixeira S. Music Therapy and Dance as Gait Rehabilitation in Patients With Parkinson Disease: A Review of Evidence. J Geriatr Psychiatry Neurol. 2019; 32(1):49-56

24. Sperens M, Georgiev D, Eriksson Domellöf M, Forsgren L, Hamberg K, Hariz GM. Activities of daily living in Parkinson's disease: Time/gender perspective. Acta Neurol Scand. 2020; 141(2):168-176.

25. Beaulieu ML, Müller MLTM, Bohnen NI. Peripheral neuropathy is associated with more frequent falls in Parkinson's disease. Parkinsonism Relat Disord. 2018; 54:46-50.

26. Carapellotti AM, Stevenson R, Doumas M. The efficacy of dance for improving motor impairments, non-motor symptoms, and quality of life in Parkinson's disease: A systematic review and meta-analysis. PLoS One. 2020; 15(8):e0236820.
27. Albani G, Veneziano G, Lunardon C, et al. Feasibility of home exercises to enhance the benefits of tango dancing in people with Parkinson's disease. Complement Ther Med. 2019; 42:233-239.

28. Bekkers EMJ, Dijkstra BW, Heremans E, Verschueren SMP, Bloem BR, Nieuwboer A. Balancing between the two: Are freezing of gait and postural instability in Parkinson's disease connected?. Neurosci Biobehav Rev. 2018; 94:113-125.

29. Cosentino C, Baccini M, Putzolu M, Ristori D, Avanzino $L$, Pelosin E. Effectiveness of Physiotherapy on Freezing of Gait in Parkinson's Disease: A Systematic Review and Meta-Analyses. Mov Disord. 2020; 35(4):523-536.

30. Kim SJ, Paeng SH, Kang SY. Stimulation in Supplementary Motor Area Versus Motor Cortex for Freezing of Gait in Parkinson's Disease. J Clin Neurol. 2018;14(3):320-326.

31. Dos Santos Delabary M, Komeroski IG, Monteiro EP, Costa RR, Haas AN. Effects of dance practice on functional mobility, motor symptoms and quality of life in people with Parkinson's disease: a systematic review with meta-analysis. Aging Clin Exp Res. 2018; 30(7):727-735

32. Michels K, Dubaz O, Hornthal E, Bega D. "Dance Therapy" as a psychotherapeutic movement intervention in Parkinson's disease. Complement Ther Med. 2018; 40:248-252.

33. Lewis C, Annett LE, Davenport S, Hall AA, Lovatt P. Mood changes following social dance sessions in people with Parkinson's disease. J Health Psychol. 2016;21(4):483492. doi:10.1177/135910531452968

34. Rocha PA, Slade SC, McClelland J, Morris ME. Dance is more than therapy: Qualitative analysis on therapeutic dancing classes for Parkinson's. Complement Ther Med. 2017; 34:1-9.

35. Kunkel D, Robison J, Fitton C, et al. It takes two: the influence of dance partners on the perceived enjoyment and benefits during participation in partnered ballroom dance classes for people with Parkinson's. Disabil Rehabil. 2018; 40(16):1933-1942.

36. Petzinger GM, Holschneider DP, Fisher BE, et al. The Effects of Exercise on Dopamine Neurotransmission in Parkinson's Disease: Targeting Neuroplasticity to Modulate Basal Ganglia Circuitry. Brain Plast. 2015; 1(1):29-39.

\section{CORRESPONDÊNCIA}

Jéssica Moratelli

Rua Juan Carlos Manucci, 88

São José - Santa Catarina

CEP 88102220, São José - Santa Catarina, Brasil.

E-mail: jessica.moratelli@hotmail.com 